\title{
ON DEVELOPABILITY
}

\author{
H. H. HUNG
}

\begin{abstract}
The converse of an old result of Bing turns out to be a very interesting characterization of developability. It is interesting in the sense that it provides a unifying framework for certain classical results of Bing, F. B. Jones, Heath and Wicke and Worrell, strengthening some of them in the process, without much effort.
\end{abstract}

In this paper, we give a conceptually simple and easily demonstrable formulation of developability, from which naturally and with ease follow certain classical results of Bing, F. B. Jones, Heath and Wicke and Worrell, in various strengths. The characterization given here (Theorem 1 ) is really the converse of a result primarily due to Bing (Theorem 9 of [1]), formulated around a notion strongly suggestive of and therefore capable of relating to (i) that of a $\sigma$-discrete network of Siwiec and Nagata [14] and therefore that of a $\sigma$-space of Okuyama [13], (ii) that of a primitive base of Wicke and Worrell [15], [16] and of course (iii) that of metrizability, especially in Bing's formulation.

Earlier ideas of Theorem 1 can be found in Green's Theorem 4.1 of [6], Burke's Theorem 2.1 of [3] and Proposition 2.1 of [4] and Brandenburg's Theorem 2.4 of [2]. We also provide a weaker characterization of developability in the end of the paper in the hope that readers may see in it even more possibilities.

THEOREM 1. A topological space $X$ is developable if there is a $\sigma$-discrete family $C=\bigcup_{n \in \mathbf{N}} C_{n}$ of closed sets, each member $C$ of which is contained in a neighbourhood $\tilde{C}$ such that if $U$ is a neighbourhood of $\xi \in X$, then there is such $a \Gamma \in C$ that $\xi \in \Gamma \subset \tilde{\Gamma} \subset U$. (And conversely.)

ProOF. Clearly, we may assume that, for all $n \in \mathbf{N}$,

$$
\tilde{C} \cap \bigcup\left(C_{n} \backslash\{C\}\right)=\emptyset, \quad \text { if } C \in C_{n}
$$

For every $n \in \mathbf{N}$, let

$$
A_{n}=\left\{\tilde{C}: C \in C_{n}\right\} \cup\left\{X \backslash \bigcup C_{n}\right\} \text {. }
$$

Noting that if $x \in C \in C_{n}$, then $\operatorname{St}\left(x, A_{n}\right)=\tilde{C}$, we can see that $A=\bigcup_{n \in \mathbf{N}} A_{n}$ is a development.

Received by the editors October 24, 1980 and, in revised form, September 24, 1981; presented to the Society, October 16, 1980 and May 26, 1981.

1980 Mathematics Subject Classification. Primary 54E30, 54E35, 54F65; Secondary 54A25, 54E20, 54E25, 54E65.

Key words and phrases. Characterization of developability, $\sigma$-discrete closed covers, neighbourhoods of $\sigma$-discrete closed sets, $\sigma$-discrete networks, point-countable bases, countable discrete families of closed sets, chrysanthemums, Moore chrysanthemums, primitive bases, symmetric neighbourhood systems, neighbourhoods of $\sigma$-conservative closed sets .

(c) 1982 American Mathematical Society 0002-9939/81/0000-0056/802.25 
Looking at Theorem 1 above, would one not ask the question that if $C$ is in place, under what circumstances can the $\tilde{C}$ 's be produced and arrive at the following theorem?

THEOREM 2. A topological space is developable if it has a $\sigma$-discrete (closed) network and a point-countable base.

Proof. Let there be a $\sigma$-discrete network $C=\bigcup_{n \in \mathrm{N}} C_{n}$ and a point-countable base $B$. Clearly, for every $C \in C$, the family

$$
B(C) \equiv\{B: B \in B, C \subset B\}
$$

is at most countable and every $C_{n}$ can be repeated countably many times to let every memeber of $B(C)$ serve as $\tilde{C}$ at least once. That if $U$ is a neighbourhood of $\xi$, there is such a $\Gamma \in C$ that $\xi \in \Gamma \subset \tilde{\Gamma} \subset U$ can be seen in the following: $B$ being a base, there is a $B \in B$ such that $\xi \in B \subset U$. $C$ being a network, there is a $C \in C$ such that $\xi \in C \subset B$, a fact ensuring that $B \in B(C)$, incidentally.

REMARK. Theorem 2 is not quite as strong as Heath's theorem that semimetrizable spaces with point-countable bases are developable [7] (see also [10]), since first countable $\sigma$-spaces are semimetrizable. But its proof is so much more immediate.

If, on the other hand, the $\tilde{C}^{\prime}$ 's are in place, we can seek to produce $C$ as follows:

THEOREM 3. A topological space with a primitive base [5], [15], [16] is developable if (and only if) it has a (countable) symmetric neighbourhood system, i.e., if the space has at every point $x$ a neighbourhood base $\left\{V_{x, n}: n \in \mathrm{N}\right\}$ such that for all $y, z$ and all $n \in \mathbf{N}, z \in V_{y, n} \Leftrightarrow y \in V_{z, n}[8]$.

ProOF. Let $\left\{W_{n}: n \in \mathbf{N}\right\}$ be a primitive base (see Definition 1.1 of [15]). For every $n \in \mathbf{N}$ and every $W \in W_{n}$, let

$$
\left.W^{k}=W \backslash \bigcup U: U<W\right\} \backslash \bigcup\left\{V_{x, k}: x \notin W\right\}, \quad \text { for every } k \in \mathbf{N} \text {. }
$$

Clearly, for every $n \in \mathbf{N}$, the family $\left\{W^{k}: W \in W_{n}, k \in \mathbf{N}\right\}$ is $\sigma$-discrete and closed and covers the space; and the family $\left\{W^{k}: W \in \mathcal{W}_{n}, k \in \mathbf{N}, n \in \mathbf{N}\right\}$ therefore fits the description of the family in the hypothesis of Theorem 1.

REMARKS. Clearly, the role played by the symmetric neighbourhood system in the theorem above can be taken up by the following requirement: On every well ordered family of open sets $(U,<)$, there are countably many functions $f^{i}$ into the collection of closed sets such that (i) $\bigcup f^{i}(W)=W \backslash \bigcup\{U: U<W\}$ and $\mathrm{Cl} \bigcup\left\{f^{i}(U): U<W\right\} \subset \bigcup\{U: U<W\}$. See also 4.3 of [16]. Incidentally, the proof of Theorem 3 above contains a proof of the converse of Theorem 1.

If 'Theorem 1 is placed beside Bing's characterization of metrizability as the availability of a $\sigma$-discrete base, one immediately sees the following:

THEOREM 4. $A T_{0}$ Moore space is metrizable if (and only if) on it (*) the union of any discrete family of closed sets can be covered by a $\sigma$-discrete family of open sets so that every member of the latter family intersects exactly one member of the former family.

REMARKs. (i) Property (*) in general does not imply normality, as the Tychonoff Plank, the most elementary and most cited counterexample of normality, would show, prompting us to wonder aloud whether we have been in some sense barking up the wrong tree when we expect solutions of the metrization problem of Moore 
spaces to be always in the form of a property somewhere between normality and collectionwise normality.

(ii) Clearly, in Theorem 4, the $\sigma$-discreteness of the covering open family postulated to exist can be weakened to $\sigma$-local finiteness without compromising the conclusion. Further, even the requirement of developability can be relaxed. Theorem 4 remains true on spaces on which there is a $\sigma$-discrete family $C=\bigcup_{n \in N} C_{n}$ of closed sets, each member $C$ of which is covered by a countable family of open sets $\tilde{C}(i)$ such that if $U$ is a neighbourhood of $\xi \in X$, then there is such a $\Gamma \in C$ that $\xi \in \Gamma \cap \tilde{\Gamma}(i) \subset \tilde{\Gamma}(i) \subset U$, for some $i$.

(iii) Theorem 4 is true among $T_{0}$ spaces, because Bing's metrization theorem is true among $T_{0}$ spaces (cf. 2.1 of [9]).

The statement of Theorem 1 permits a direct derivation of the following classical result of $\mathrm{F}$. B. Jones [12].

THEOREM 5. Developable spaces on which all discrete families of closed sets are countable are second countable (and therefore metrizable if they are also $T_{3}$ ).

To further exploit Theorem 1, we apply it to the chrysanthemum [11] which is defined as follows.

DEFINITION. A topological space $X$ is a chrysanthemum, if $X=\bigcup\left\{A_{\xi}: \xi<\right.$ $\alpha$ for some ordinal $\alpha$ such that (i) there is such a closed subset $A \subset X$ that (a) $A_{\xi} \backslash A$ is open, for all $\xi<\alpha$, and (b) $A_{\xi} \cap A_{\eta}=A$, for all $\xi \neq \eta, \xi, \eta<\alpha$; and (ii) on every $A_{\xi}$, discrete families of closed subsets are countable.

REMARKS. All hereditarily separable spaces and, in particular, separable metric spaces are chrysanthemums, as are all Lindelöf spaces and all countably compact spaces. Kowalsky's hedgehogs are also chrysanthemums. For the purpose of the present application, the following additional property on the chrysanthemum is useful:

(iii) every neighbourhood of $A$ contains within it a closed neighbourhood of $A$, a property also shared by all the spaces mentioned above.

THEOREM 6. T0 Moore chrysanthemums with property (iii) are metrizable (and all metrizable spaces are subspaces of countable products of such spaces).

Proof. Let $X=\bigcup\left\{A_{\xi}: \xi<\alpha\right\}$ be a chrysanthemum, such that (i), (ii) and (iii) above are true. Let there be a $\sigma$-discrete family $C=\bigcup_{n \in N} C_{n}$ of closed sets of the description of that in Theorem 1. For every $m \in \mathbf{N}$, let

$$
D_{m}=\left\{C \in C_{m}: C \cap A \neq \emptyset\right\}, \quad \text { and } \quad \varepsilon_{m}=C_{m} \backslash D_{m} .
$$

Clearly $D_{m}$ is countable and can be ordered into

$$
\left\{D_{m, 1}, D_{m, 2}, \ldots\right\} \text {. }
$$

Clearly also, we can assume that $C \subset A_{\xi}$, if $C \in \mathcal{E}_{m}$ and $C \cap A_{\xi} \neq \emptyset$; and, for every $\xi<\alpha$,

$$
\mathcal{E}_{m}(\xi) \equiv\left\{C: C \in \mathcal{E}_{m}, C \subset A_{\xi}\right\}
$$

is countable and can be ordered into

$$
\left\{E_{m}(\xi, 1), E_{m}(\xi, 2), \ldots\right\} .
$$


For every $j \in \mathbf{N}$, the set $X \backslash \bigcup_{\xi<\alpha} E_{m}(\xi, j)$ being an open neighbourhood of $A$, there is a closed neighbourhood $G_{m, j}$ of $A$ within it. If we let

$$
F_{m}(\xi, j) \equiv\left(\tilde{E}_{m}(\xi, j) \cap\left(A_{\xi} \backslash A\right)\right) \backslash G_{m, j}
$$

we have

$$
\mathcal{F}_{m, j} \equiv\left\{F_{m}(\xi, j): \xi<\alpha\right\}
$$

which is discrete, because there is an open cover (of $X$ )

$$
\left\{\left(A_{\xi} \backslash A\right): \xi<\alpha\right\} \cup\left\{\operatorname{Int} G_{m, j}\right\}
$$

each memeber of which intersects at most one member of $\mathcal{F}_{m, j}$; and therefore a $\sigma$-discrete base for $X$ in the following family:

$$
\left\{\mathcal{F}_{m, j}: m, j \in \mathbf{N}\right\} \cup\left\{\left\{\tilde{D}_{m, j}\right\}: m, j \in \mathbf{N}\right\},
$$

and consequently the metrizability of $X$.

In the way that Theorem 2.1 of [9] generalizes Bing and many others simultaneously, we can generalize Theorem 1 to Theorem 7 below that is redolent of Brandenburg, in the hope that readers may see in it even more possibilities.

THEOREM 7. A topological space $X$ is developable if there is a $\sigma$-conservative (i.e. $\sigma$-closure-preserving) family $C=\bigcup_{n \in \mathrm{N}} C_{n}$ of closed sets, each member $C$ of which is contained in a neighbourhood $\tilde{C}$ such that, for every $\xi \in X$,

$$
\left\{\bigcap\left\{\tilde{C}: \xi \in C \in C_{n}\right\}: \xi \in \bigcup C_{n}\right\}
$$

is a neighbourhood base.

ProOF. Let there be given a $\sigma$-conservative $C=\bigcup_{n \in \mathbb{N}} C_{n}$ of closed sets of the description above. For every $x \in X$ and every $n \in \mathbf{N}$, mindful that $\cap \emptyset=X$, let

$$
U(x, n) \equiv \operatorname{Int} \bigcap\left\{\tilde{C}: x \in C \in C_{n}\right\} \backslash \bigcup\left\{C: x \notin C \in C_{n}\right\} .
$$

Noting that, for every $\xi \in X$, if $\xi \in U(x, n)$, then $x \in C \in C_{n}$ when $\xi \in C$ and consequently $U(x, n) \subset \operatorname{Int} \bigcap\left\{\tilde{C}: \xi \in C \in C_{n}\right\}$, we can see that

$$
\bigcup_{n \in \mathbb{N}}\{U(x, n): x \in X\}
$$

is a development of $X$.

\section{REFERENCES}

1. R. H. Bing, Metrization of topological spaces, Canad. J. Math. 3 (1951), 175-186.

2. H. Brandenburg, Some characterizations of developable spaces, Proc. Amer. Math. Soc. 80 (1980), 157-161.

3. D. K. Burke, Preservation of certain base axioms under a perfect mapping, Topology Proc. 1 (1976), 269-279.

4. __ Pseudo-open mappings from topological sums, Proc. Amer. Math. Soc. 74 (1979), 191-196.

5. D. K. Burke and D. J. Lutzer, Recent advances in the theory of generalized metric spaces, Topology (Proc. Ninth Annual Spring Topology Conf., Memphis State Univ., Memphis, Tenn., 1975), Lecture Notes in Pure and Appl. Math., Vol. 24, Dekker, New York, 1976, pp. 1-70.

6. J. W. Green, Completion and semicompletion of Moore spaces, Pacific J. Math. 57 (1975), 153165.

7. R. W. Heath, On spaces with point-countable bases, Bull. Acad. Polon. Sci. Sér. Sci. Math. Astronom. Phys. 13 (1965), 393-395. 
8. H. H. Hung, One more metrization theorem, Proc. Amer. Math. Soc. 57 (1976), 351-353.

9. __ A contribution to the theory of metrization, Canad. J. Math. 29 (1977), 1145-1151.

10. __ A note on a theorem of Heath, Topology Appl. (to appear).

11. Collectionwise normality and fragmented collectionwise normality-Applications, Abstracts Amer. Math. Soc. 2 (1981), 410.

12. F. B. Jones, Concerning normal and completely normal spaces, Bull. Amer. Math. Soc. 43 (1937), 671-677.

13. A. Okuyama, $\sigma$-spaces and closed mappings. I and II, Proc. Japan Acad. 44 (1968), $472-481$.

14. F. Siwiec and J. Nagata, A note on nets and metrization, Proc. Japan Acad. 44 (1968), 623-627.

15. H. H. Wicke, A functional characterization of primitive base, Proc. Amer. Math. Soc. 72 (1978), 355-361.

16. H. H. Wicke and J. M. Worrell, Jr., A characterization of spaces having bases of countable order in terms of primitive bases, Canad. J. Math. 27 (1975), 1100-1109.

Department of MAthematics, MCGill University, Montrétal, Québec, CANADA H3A 2K6 